\title{
Attention need not always apply: Mind wandering impedes explicit but not implicit sequence learning
}

\author{
Nicholaus P. Brosowsky ${ }^{1}$, Samuel Murray $^{1}$, Jonathan Schooler ${ }^{2}, \&$ Paul Seli $^{1}$ \\ ${ }^{1}$ Department of Psychology and Neuroscience, Duke University, Durham, NC, USA \\ ${ }^{2}$ Department of Psychological and Brain Sciences, University of California Santa Barbara, Santa Barbara, CA, USA
}

\begin{abstract}
Preprint
According to the attentional resources account, mind wandering (or "task-unrelated thought") is thought to compete with a focal task for attentional resources. Here, we tested two key predictions of this account: First, that mind wandering should not interfere with performance on a task that does not require attentional resources; second, that as task requirements become automatized, performance should improve and depth of mind wandering should increase. Here, we used a serial reaction time task with implicit- and explicit-learning groups to test these predictions. Providing novel evidence for the attentional resource account's first prediction, results indicated that depth of mind wandering was negatively associated with learning in the explicit, but not the implicit, group, indicating that mind wandering is associated with impaired explicit, but not implicit, learning. Corroborating the attention resource account's second prediction, we also found that, overall, performance improved while at the same time depth of mind wandering increased. From an implicitlearning perspective, these results are consistent with the claim that explicit learning is impaired under attentional load, but implicit learning is not. Data, analysis code, manuscript preparation code, and pre-print available at osf.io/qzry7/.
\end{abstract}

Keywords: mind wandering, inattention, implicit learning, serial reaction time task Word count: 6810

From time to time, you might find that your mind wanders away from a task toward something unrelated. For instance, perhaps while driving to work, you catch yourself thinking about the groceries you need to get later in the day, or the upcoming fishing trip that you have planned for the weekend. Of course, failing to maintain your attention on the road can be dangerous, increasing the likelihood you will make a potentially disastrous mistake (Gil-Jardiné et al., 2017; Yanko \& Spalek, 2014). Alas, mind wandering tends to get a bad rap for its often-negative consequences on task performance (see Mooneyham \& Schooler, 2013 for a review); indeed, some even refer to it as a curse (Hasenkamp, 2013)! Nevertheless, mind wandering can have positive consequences. For instance, returning to our opening example, mind wandering could help you plan the next grocery shopping trip, help you generate a creative recipe (i.e., future planning and creativity; Smallwood \& Schooler, 2015), or provide a "mental break" from the mind-numbing activity of driving (i.e., attentional dishabituation; Mooneyham \& Schooler, 2016).

Although several accounts have been offered to explain how mind wandering can have these distinctive positive and negative effects (e.g., McVay \& Kane, 2010; Seli, Kane, et al., 2018; Seli, Risko, Smilek, \& Schacter, 2016; Smallwood, 2013; Smallwood \& Schooler, 2015), arguably, the most popular of these is the attentional resources account. On this view, mind wandering-hereafter conceptualized as task-unrelated thought (Seli, Kane, et al., 2018)—and task-relevant processes compete for attentional resources (Smallwood \& Schooler, 2006).

The attentional resources account makes several predictions about the relationship between mind wandering and task engagement. First, it predicts that if mind wandering and task engagement draw on the same attentional resources, then focusing on a task (i.e., allocating attentional resources to task performance) should result in lower rates of mind wandering, as there are fewer resources available for mind wandering. Second, and relatedly, this account predicts that more mind wandering should diminish task performance when both mind wandering and task engagement draw on the same resources. Third, if mind wandering and task engagement do not compete for attentional resources, then mind wandering should not impair task performance. In other words, if mind wandering and task engagement do not draw on the same resources, then the processes supporting each can run in parallel and should not interfere with 
each other. Finally, the attentional resources account predicts that mind wandering should increase as a function of task automatization—or by proxy, time-on-task (Antrobus, 1968; Cunningham, Scerbo, \& Freeman, 2000; Smallwood et al., 2004; Smallwood, Baracaia, Lowe, \& Obonsawin, 2003). Specifically, as time-on-task increases, people learn the task and subsequently automatize elements of task performance (Logan, 1988; Logan \& Compton, 1998; Moors \& De Houwer, 2006; Newell \& Rosenbloom, 1981; Schneider \& Shiffrin, 1977; Shiffrin \& Schneider, 1977). Automatization results in fewer resources needed to maintain task performance, which leaves more resources available for mind wandering (Smallwood \& Schooler, 2006). Thus, the increase in mind wandering with time-on-task corresponds to the rate of learning in the focal task, or task automatization.

Research on mind wandering provides abundant support for the first two predictions. Increased task focus (resulting from increases in task difficulty) typically decreases the rate of mind wandering (e.g., Brosowsky, Murray, Schooler, \& Seli, under review-b; Giambra, 1989; Seli et al., 2018; Smallwood et al., 2011; Thomson, Besner, \& Smilek, 2013; Xu \& Metcalfe, 2016). Research has also found that mind wandering impairs task performance in a variety of contexts (e.g., Franklin, Smallwood, Zedelius, Broadway, \& Schooler, 2016; Reichle, Reineberg, \& Schooler, 2010; Seli, Cheyne, \& Smilek, 2013; J. Smallwood et al., 2008a, 2008b; Smallwood et al., 2004).

Surprisingly, there is little work investigating the third prediction of the attentional resource account: that some tasks can run in parallel with mind wandering without interference. This might be due to the outsized use of attentionally demanding tasks in mind-wandering research, which are likely to compete for attentional resources with mind wandering. As such, it is perhaps unsurprising that the vast majority of the literature has found that mind wandering impairs task performance (and, likewise, unsurprising that mind wandering has earned a bad reputation). Indeed, if people perform an attentionally demanding task without focusing on it (i.e., they are mind wandering), probably their mind wandering will be associated with poorer task performance.

Mountains of evidence conflict with the fourth prediction of the attentional resources account: that task automatization is associated with increases in mind wandering. Although mind wandering typically does increase as a function of time-on-task (Antrobus, 1968; Cunningham et al., 2000; Smallwood et al., 2004, 2003), this increase does not appear to reflect automatization of primary-task performance. Indeed, one implication of this prediction is that task automatization should reflect stable (if not improved) performance over time. However, as mentioned previously, many studies find that performance decreases as a function of time-on-task (e.g., Brosowsky, Degutis, Esterman, Smilek,
\& Seli, in press-a; Krimsky, Forster, Llabre, \& Jha, 2017; McVay \& Kane, 2012; Metcalfe \& Xu, 2016; Risko, Anderson, Sarwal, Engelhardt, \& Kingstone, 2012; Teasdale et al., 1995; Thomson, Seli, Besner, \& Smilek, 2014).

The conflicting evidence, however, should be taken with a grain of salt: Tasks used to study mind wandering (e.g., SART, vigilance tasks, metronome response tasks) are unlikely to be appropriate for evaluating the relationship between learning, skill-acquisition (i.e., task automatization) and mind wandering. These tasks are simple and monotonous by design, often requiring single-button responses, and presenting target stimuli that are either rare and unpredictable (e.g., SART) or perfectly predictable (e.g., the metronome response task). In other words, such tasks are unsuitably structured for learning because there is nothing to learn about the tasks beyond their very basic performance requirements. Likewise, there are few, if any, skills to develop while performing such tasks. In these situations, then, mind wandering is less likely to reflect learning than it is to reflect a scenario in which participants give up on fully focusing on the task (Brosowsky et al., in press-a; Seli et al., 2013).

The attentional resources account predicts that increased learning should lead to increased mind wandering by "freeing up" resources to be allocated to mind wandering. Critically, however, this argument presumes that tasks can be learned; while this may be true, the tasks employed in many laboratory studies tend not to permit such learning. Moreover, in light of restricting mind-wandering research to a few attentionally demanding tasks, extant research has not investigated whether mind wandering can run in parallel with certain kinds of tasks. Thus, to test these two predictions of the attentional resources account, different tasks must be used.

Therefore, in the current study, we assessed whether (a) a focal task that does not draw on the same attentional resources as mind wandering can run in parallel with mind wandering without interference, and (b) taskautomatization is associated with increases in mind wandering. To do this, we examined the influence of mind wandering on performance during an implicit (versus explicit) sequence-learning task.

Implicit learning is the process of acquiring information about the underlying structure of the material without any intention to do so (Keele, Ivry, Mayr, Hazeltine, \& Heuer, 2003; Nissen \& Bullemer, 1987; Reber, 1989; Seger, 1994). Implicit learning occurs across a variety of domains (Cleeremans, Destrebecqz, \& Boyer, 1998), but is most commonly investigated using a serial reaction time (SRT) task (Nissen \& Bullemer, 1987; for a review, see Schwarb \& Schumacher, 2012). In this task, a target can appear in one of four locations, and participants are instructed to identify the location of a target by pressing a corresponding key. 
Critically, unbeknownst to participants, targets occur in a fixed sequence that repeats throughout the experiment until eventually the sequence changes to a new sequence of target locations. Participants typically perform better on the training sequence as compared to the unpracticed sequence. However, despite this performance advantage, participants are often unable to reconstruct the sequence, and they report no awareness of the repeating sequence, which rules out explicit learning (Pasquali, Cleeremans, \& Gaillard, 2019; Schwarb \& Schumacher, 2012).

Historically, there has been considerable debate as to whether implicit learning relies on attentional resources (Cleeremans, Allakhverdov, \& Kuvaldina, 2019; Jiménez, 2003). Nevertheless, numerous results converge on the view that implicit learning does not require attentional resources (e.g., Baker, Olson, \& Behrmann, 2004; Frensch \& Miner, 1995; Jiang \& Chun, 2001; Jiménez \& Mendez, 1999; TurkBrowne, Jungé, \& Scholl, 2005). Explicit learning, however, requires executive processing and attentional resources to guide attention, maintain task goals, and reduce interference (e.g., Cowan, 1988; Frensch \& Miner, 1995; Johnson \& Hirst, 1993; Unsworth \& Engle, 2005). Therefore, the SRT task is well-suited for our present goals because it allows us to examine the influence of mind wandering during identical tasks with and without attentional resource demands. Furthermore, this task permits us to measure changes in task automatization over time, and it is sensitive to small variations in learning rates via multiple measures: the improvement in performance over time and the disruption caused by changing to an unlearned sequence (i.e., "transfer" effects; Pasquali et al., 2019).

Practice effects-the gradual reduction in reaction times over time-reflect participants' growing expertise in performing task. However, practice effects also include learning about non-sequence task information like visuo-motor associations or stimulus-response mappings and may be contaminated by other factors such as fatigue or motivation. As such, it has become commonplace to measure learning using transfer effects-the change in performance that occurs when a new sequence is presented in place of the trained sequence (e.g., Cohen, Ivry, \& Keele, 1990; Keele, Jennings, Jones, Caulton, \& Cohen, 1995; Schumacher \& Schwarb, 2009; Willingham, Nissen, \& Bullemer, 1989). If participants have automatized the trained sequence then we would expect them to perform worse (e.g., slower reaction times, more errors) when a new sequence is presented after practicing the trained sequence as participants no longer benefit from the knowledge of the underlying sequence. Thus, the size of the transfer effect indexes the amount of sequence-specific learning.

Notably, one prior study investigated the effect of mind wandering on performance on the SRT task (Franklin et al., 2016). While this study found—at odds with the attentional resource account - that mind wandering interfered with SRT task performance, there are several limitations of this study that bear mentioning.

First, the study did not include an explicit (intentional) learning group for comparison. Critically, however, such a comparison allows researchers to test for (a) poorer performance and (b) less explicit knowledge in the implicit relative to the explicit learning group, both of which outcomes allow one to more confidently conclude that implicit learning has in fact taken place.

Second, although the measurement of awareness in SRT tasks is still a contentious issue (Pasquali et al., 2019; Schwarb \& Schumacher, 2012), Franklin et al. (2016) employed a now-outdated and atypical measure of awareness that has since been replaced with improved measures (Pasquali et al., 2019). More specifically, Franklin et al. used a version of the "inclusion generation" task, which requires participants to reproduce the training sequence from memory. Importantly, this inclusion generation task fails to adequately dissociate implicit and explicit learning because, during this task, participants who implicitly learn a sequence can often still generate the sequence above chance (see Pasquali et al., 2019). In light of these shortcomings, most contemporary studies employ an "exclusion generation task," which requires participants to generate the training sequence in reverse. Critically, this exclusion generation task (which was not employed in the Franklin et al. study) allows one to more confidently dissociate implicit and explicit learning because it requires participants to mentally rehearse and reverse the sequence, a task that requires explicit knowledge of the sequence. Thus, when participants can generate patterns from the reverse sequence above chance, one can more confidently say they had acquired explicit knowledge.

The final limitation of Franklin et al.'s (2016) study is that the authors alternated the training sequence and random sequences in a predictable fashion (two random, six training, two random, etc.) throughout the entire task and, critically, they did not report how performance changed across the experiment; instead, they reported an aggregate of early performance (presumably prior to any learning) and later performance (after learning had presumably occurred). If, as the authors argue, the modest performance benefit for training sequences occurred because participants learned the sequence, then the difference in performance between the training and random sequences should have increased over time (since more learning would take place with increased exposure to the learning sequence). However, because Franklin et al.'s performance measure was an aggregate of early and later performance, this essential test of implicit learning is absent from their article. Consequently, the reader is unable to assess how-or ifparticipants were learning, and to what extent mind wander- 
ing influenced learning rates (if learning did in fact occur). Therefore, Franklin et al.'s results cannot directly speak to the hypotheses proposed by the attentional resources account, which require an assessment of performance and mind wandering over time.

One important methodological note worth discussing is the use of online data collection procedures ${ }^{1}$. To our knowledge, there is only one study that has validated the SRT task in an online setting (Sævland \& Norman, 2016). In their study, using an alternating SRT task, the researchers found that both learning and sequence-generation performance was comparable across in-lab and online samples. This is consistent with a larger body of research demonstrating that data collected from online samples (e.g., Amazon Mechanical Turk) are comparable to convenience samples like undergraduate students (e.g. Armitage \& Eerola, 2020; Buhrmester, Talaifar, \& Gosling, 2018; Casler, Bickel, \& Hackett, 2013; Dandurand, Shultz, \& Onishi, 2008; Hilbig, 2016; Leeuw \& Motz, 2016), and consistent with numerous studies that have successfully implemented different reaction time tasks online [e.g., rhythmic tapping tasks, Brosowsky et al. (in press-a); Seli et al. (2013); visual selective attention tasks, Bejjani, Tan, and Egner (2020); Brosowsky and Crump (2020); Brosowsky and Crump (2018); typing tasks, Behmer Jr and Crump (2016); Crump, Lai, and Brosowsky (2019); psycholinguistic tasks, Kim, Gabriel, and Gygax (2019); statistical learning, Dale, Duran, and Morehead (2012); n-back working memory tasks, Brosowsky et al. (under review-b); Smith, Brosowsky, Daniel, Meier, and Seli (under review); to name a few].

However, controversy surrounding the quality of online data persists (e.g., Chmielewski \& Kucker, 2020). Therefore, a secondary aim of the current study was to replicate Pasquali et al. (2019) and further validate the usage of online procedures to measure implicit learning (e.g., Sævland, et al., 2016). To address potential data quality concerns, we collected data from a much larger sample than necessary. Indeed, Pasquali et al. observed a $193 \mathrm{~ms}$ ( $\mathrm{sd}=79 \mathrm{~ms}$ ) and $249 \mathrm{~ms}$ ( $\mathrm{sd}=114 \mathrm{~ms}$ ) transfer effect for the implicit and explicit groups, respectively (a $\sim 60 \mathrm{~ms}$ difference between groups). Using a monte-carlo simulation procedure (see Brosowsky \& Crump, 2020; Crump, Brosowsky, \& Milliken, 2017) and the reaction time distributions from Pasquali et al., we estimated at least 45 participants per group would be required to reliably detect a $60 \mathrm{~ms}$ difference with $80 \%$ power. However, we opted to collected data from 100 participants in each group so that we could reliably detect a 60 ms difference between groups with $99 \%$ power; a difference of $50 \mathrm{~ms}$ with $95 \%$ power; and a difference as small as $40 \mathrm{~ms}$ with $82 \%$ power.

\section{The Present Study}

In the present study, participants completed a fiveresponse serial reaction time task. On every trial, participants responded to one of five targets, each corresponding to a response on the keyboard. Participants practiced a 10-element repeating sequence for 13 blocks of 80 trials before switching to a new, unpracticed sequence in the 14th block, and ending with the training sequence in the 15 th block. To assess awareness, we had participants complete two sequence generation tasks: the inclusion task, wherein participants generate the training sequence for 100 trials, and the exclusion generation task, wherein participants generate the training sequence in reverse (Pasquali et al., 2019). Whereas participants in the "explicit" group were given instructions about memorizing the repeating sequence, participants in the "implicit" group were not given any information about the sequences.

Our design addresses the aforementioned limitations with Franklin et al.'s (2016) study in several important ways: first, we measured both mind wandering and task performance as a function of time on task to determine whether mind wandering is associated with task automatization. To assess changes in learning and depth mind wandering we will measure "practice" and "transfer" effects. Practice effects index the change in performance that occurs as participants gain experience with the task. Specifically, we will compare performance and depth of mind wandering in block 13 to block 1 . Transfer effects index the change in performance that occurs when the sequence is changed (i.e., whether participants' current performance/depth of mind wandering "transfers" to a block with a new sequence). Here, we will compare performance and depth of mind wandering in block 14 (the reversed block) to blocks 13 and 15 . Second, we included both explicit and implicit learning groups, allowing us to draw direct comparisons between these groups and to determine whether implicit learning (as compared to explicit learning) is negatively associated with mind wandering. Third, we employed more robust measures of awareness, which allowed us to more confidently rule out explicit learning in the implicit group.

\section{Method}

We report all data exclusions and all measures in the study. Moreover, in accordance with the recommendations of Seli, Kane, et al. (2018), we report that, in the present study, we conceptualized mind wandering as task-unrelated thought, and we operationally defined it for our participants in terms of thoughts pertaining to something other than what they were doing when queried about their mental states (see below for more details). All data, analysis, experiment, and manuscript preparation code can be found can

\footnotetext{
${ }^{1}$ We thank Dr. Jiménez for drawing our attention to this issue.
} 
A.

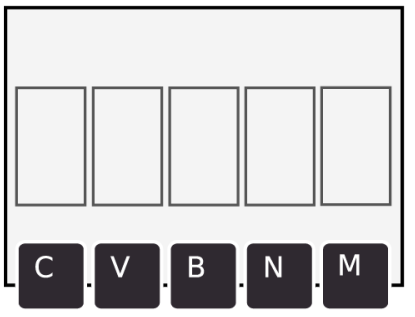

B.

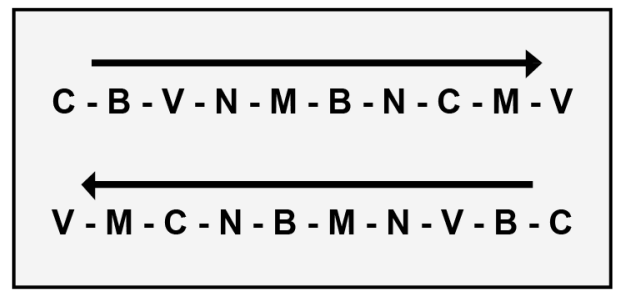

C.

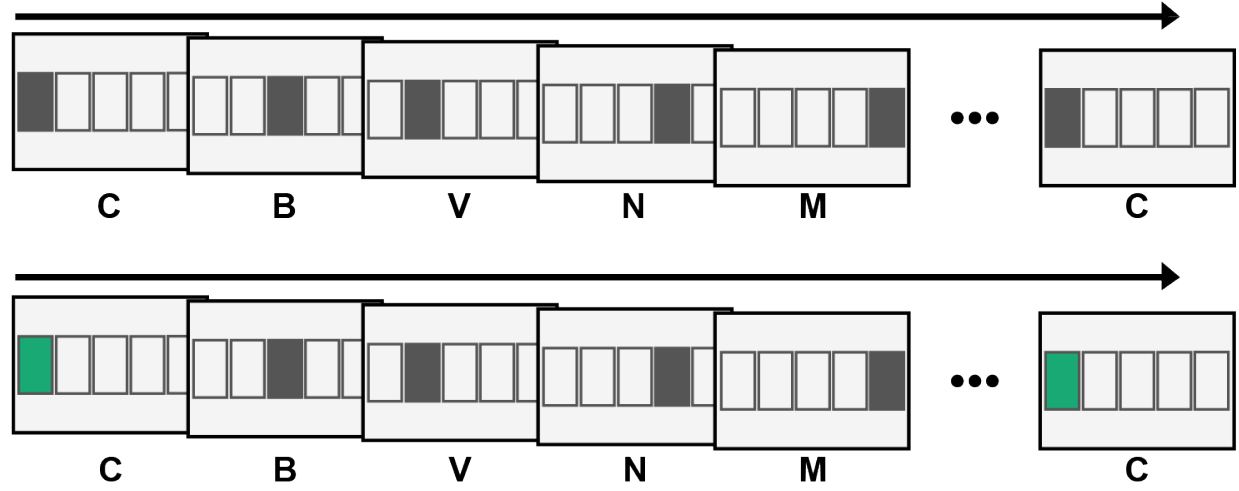

Figure 1. Illustrations of the task and stimuli used. Figure A illustrates the keyboard responses associated with each of the five possible target locations. Figure B shows an example of the training sequence and the corresponding reverse sequence (Pasquali et al., 2019). Each participant was randomly assigned a sequence from the total set of 840. Participants practiced the training sequence for 13 blocks before switching to the reverse sequence for the 14th block and returning to the training sequence in the 15th block. Figure $\mathrm{C}$ illustrates a series of trials for the implicit group (above) and the explicit group (below). Stimulus presentation differed only in that the first target in the sequence was presented in green for the explicit group.

be found at osf.io/qzry7/.

\section{Participants}

Participants were 200 individuals $^{2}$ who completed a Human Intelligence Task (HIT) posted on the Amazon Mechanical Turk. Participants were paid \$3.25 (U.S. dollars) for completing the HIT, which lasted approximately 25 minutes. Participants were told that the experiment would take fewer than 30 minutes to complete but were not informed about how many trials they would be presented or how often they would be asked to report their thoughts.

\section{Serial Reaction Time (SRT) Task}

The primary task was a serial reaction time (SRT) task, with five response options, each corresponding to a single location (see Figure 1). The display contained five rectangles, each corresponding to a response on the keyboard (left to right: $\mathrm{C}, \mathrm{V}, \mathrm{B}, \mathrm{N}$, and $\mathrm{M}$ ), displayed in dark grey on an off-white background. Each rectangle was 125.6 x 196 pixels and displayed 14.4 pixels apart. Each rectangle was initially outlined in dark grey and filled with off-white. On each trial, one rectangle changed color (dark grey or green) and participants were instructed to press the corresponding key as quickly and as accurately as possible. Once the participant pressed the correct key, the next target would immediately appear. Participants received the following instructions:

In this part of the experiment, you will be presented five rectangles in a row. On each trial, one of the rectangles will be filled with a color and you will respond by pressing one of five corresponding keys on the keyboard as quickly and as accurately as possible."

[example of stimulus display]

You will use the "C", "V", "B", "N", and "M" keys on the keyboard, each corresponding to one of the five rectangles from left to right: $\mathrm{C}-\mathrm{V}-\mathrm{B}-\mathrm{N}-$ $\mathrm{M}$. You will get the next rectangle after you've responded correctly.

\footnotetext{
${ }^{2}$ Due to a programming oversight, we did not collect any demographic information.
} 
For example, if you were presented:

[example of stimulus display]

You would respond " $\mathrm{V}$ " because it is the second from the left rectangle (C-V-B-N-M).

And if you were presented:

[example of stimulus display]

You would respond " $\mathrm{M}$ " because it is the fifth from the left rectangle (C-V-B-N-M)

[Only for the explicit condition] Important: the same sequence of locations/responses will repeat throughout the experiment. You will know when you are at the beginning of the sequence because the color of the rectangle will be green instead of black:

[example of stimulus display]

Your task for this experiment is to try to respond as quickly as and as accurately as possible.

[Only for the explicit condition] As you complete the task you should try to memorize the repeating sequence so that you can respond as quickly as possible.

The experiment followed a typical SRT paradigm (see Pasquali et al. 2019): Participants completed 13 blocks of the training sequence, followed by a transfer block containing a new sequence (block 14), followed by a return to the original sequence (block 15). The entire experiment consisted of 15 blocks of 80 trials (1200 trials). Blocks 1 through 13 and block 15 contained a repeating 10-element sequence, referred to as the "training sequence". The 14th block consisted of a new sequence, which was a reversal of the original, training sequence (i.e., the "reverse sequence").

We used 10-element refined reversible second-order conditional (RSOC) sequences as described by Pasquali et al. [2019; see also (Jiménez, Méndez, Pasquali, Abrahamse, \& Verwey, 2011). These are second-order conditional sequences that are traditionally used in SRT tasks. Each target appears with the same frequency-twice per sequence loop-and each transition occurs with equal frequency. In addition, refined RSOC sequences contain no common transitions with their reversed sequence patterns (i.e., "reversible"), but are otherwise fully analogous to the original sequence. Finally, refined RSOC sequences do not contain any ascending or descending runs (i.e., "refined"). Ascending and descending runs are excluded to eliminate potential abstract cues and keystroke facilitations that may enable chunking strategies (abstractly homogeneous; "no transition would be responded faster than others in the absence of learning"; cf., Jiménez, 2008). Each participant was randomly assigned a refined RSOC sequence from the total set of 840 .
Participants were either assigned to the implicit (incidental) or explicit (intentional) learning group. The task and instructions were identical for both groups with two exceptions: First, in the explicit learning group, a green rectangle appeared in place of the black rectangle. This was to indicate the beginning of the sequence (no green rectangle appeared in the transfer block, but participants were not told in advance about the change in task). Second, participants in the explicit group also received the following additional instructions:

Important: the same sequence of locations/responses will repeat throughout the experiment. You will know when you are at the beginning of the sequence because the color of the rectangle will be green instead of black.

As you complete the task you should try to memorize the repeating sequence so that you can respond as quickly as possible.
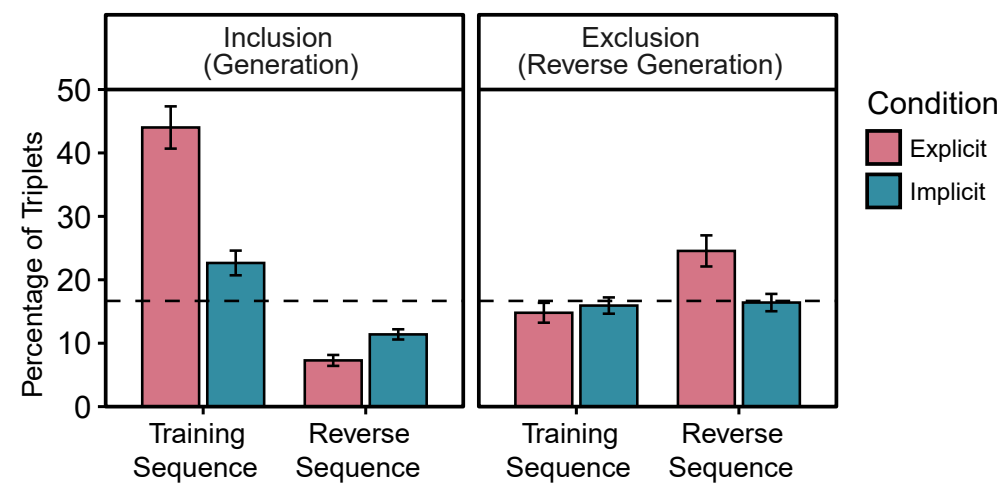

Figure 2. Results of the sequence generation tasks used to assess awareness across the explicit and implicit learning groups. Plots show the percentage triplets produced that match triplets from the training or reverse sequence when asked to produce the training sequence (inclusion generation task) or the reverse sequence (exclusion generation task).

\section{Generation Tasks}

After the SRT task, awareness was assessed with two unexpected sequence generation tasks. In the inclusion generation task, participants were instructed to generate the training sequence over 100 keypresses (if performed correctly, this would correspond to generating the full sequence 10 times). The display began with one of the five rectangles randomly filled, and participants were instructed to press the button corresponding to the next location that would have appeared in the training sequence and to continue with the sequence for 100 keypresses. Participants were given the following instructions: 
Now we are going to test your knowledge of the sequence you've been practicing.

In this part of the experiment, we want you to generate the practiced sequence from memory for 100 trials.

The session will begin at a random location. You will respond with the next location that would have come right after it in the sequence you have practiced. Then you will respond again with the location that would come next, and so on, generating the sequence you have practiced.

If you are unsure of the next location, try to rely on your intuition and respond as best as you can.

The display will be identical to the practice and the boxes will change color based on your response.

The instructions differed slightly for the implicit learning group. For them, we included an additional statement to assure participants that they were not required to learn the sequence to receive payment ("You may or may not have noticed that there was a sequence of locations/responses that repeated throughout the experiment (if you didn't notice, that's ok)").

Participants then completed the exclusion generation task. This was the same as the inclusion task, except here participants were instructed to generate the reverse sequence from the one they had practiced:

In this part of the experiment, we want you to generate the reversed pattern of the previously learned sequence from memory for 100 trials.

The session will begin at a random location. You will respond with the location that would have come before it in the sequence. After that, you will respond again with the one that would have come before that, and so on, generating the sequence in reverse.

If you are unsure of the next location, try to rely on your intuition and respond as best as you can.

The display will be identical to the practice and the boxes will change color based on your response.

Following the procedure outlined by Pasquali et al. (2019), performance on the generation tasks was assessed by computing the number of training and reverse sequence triplets. The number of triplets we expected they would produce by chance alone was computed as the number of triplets in the sequence divided by the total number of possible triplets; that is, $10 /(5 \times 4 \times 3)=16.667 \%$.

\section{Thought probes}

Throughout the SRT task, depth of mind wandering was sampled using intermittently presented thought probes. Thought probes were presented at the end of every block of 80 trials. When a thought probe was presented, the task temporarily stopped, and the participant was presented with the following question: "To what extent were you mind wandering?" Participants were instructed to report their depth of mind wandering by using a sliding scale, the anchors for which were "Not at all Mind Wandering" (which corresponded with a value of 0 ) and "Fully Mind Wandering" (which corresponded with a value of 100).

At the beginning of the experiment, participants were given the following instructions, followed by a working example of the thought probe response interface:

While you are completing this task, you may find yourself thinking about things other than the task. These thoughts are referred to as "taskunrelated thoughts" or "mind wandering". Having task-unrelated thoughts is perfectly normal, especially when one must do the same thing for a long period of time.

We would like to determine how frequently you were thinking about the task versus how frequently you are thinking about something unrelated to the task (mind wandering). To do this, every once in a while, the task will temporarily stop and you will be presented with a thoughtsampling screen that will ask you to indicate to what extent you have been focused on the task (not at all mind wandering) or focused on taskunrelated thoughts (fully mind wandering).

Being focused on the task means that you were focused on some aspect of the task at hand. For example, if you have been thinking about your performance on the task, or about when you should make a button press, these thoughts would count as being on-task.

On the other hand, experiencing task-unrelated thoughts means that you were thinking about something completely unrelated to the task. For example, thinking about what to eat for dinner, about an upcoming event, or about something that happened to you earlier in the day. Any thoughts that you have that are not related to the task you are completing count as task unrelated. 

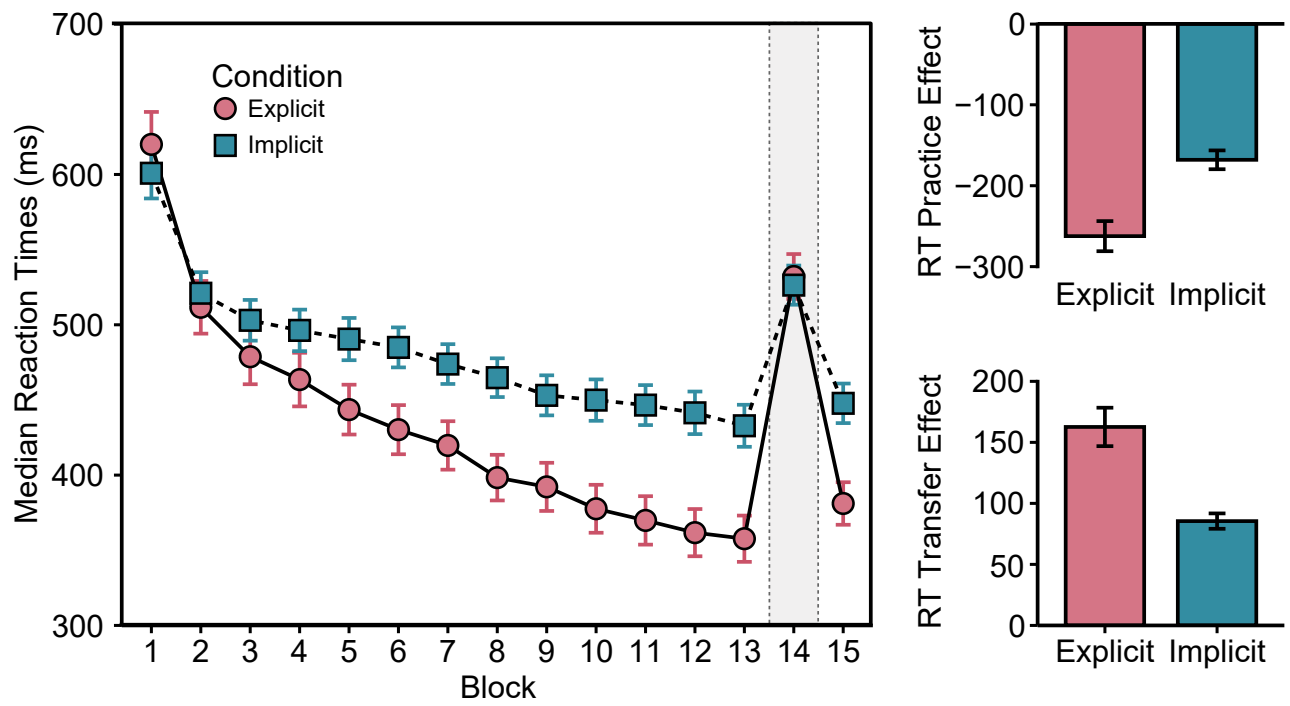

Figure 3. Results of the serial reaction time task. Median reaction times are plotted across trial blocks on the left. Practice effects (reaction times in the 1st block are subtracted from the 13th block) and transfer effects (reaction times in the 14th block are subtracted from the average of the 13th and 15th blocks) are plotted on the right.

When the thought-sampling screen is presented, we will ask you to indicate the extent to which you have been mind wandering. You will indicate the extent you have been mind wandering on a scale from 0 to 100 (0 being not at all mind wandering, 100 being fully mind wandering).

\section{Data analysis and manuscript preparation}

This manuscript was prepared using $\mathrm{R}$ ( $\mathrm{R}$ Core Team, 2019). A variety of notable $R$ packages were used for data analysis (Bates, Mächler, Bolker, \& Walker, 2015; Fox \& Weisberg, 2019; Kuznetsova, Brockhoff, \& Christensen, 2017; Singmann, Bolker, Westfall, Aust, \& Ben-Shachar, 2019; Wickham et al., 2019; Wickham \& Henry, 2019), data visualization (Fox \& Weisberg, 2018; Kassambara, 2019; Wickham, 2016; Wilke, 2019), and general manuscript preparation (Aust \& Barth, 2018). All data, analysis, and manuscript preparation code can be found at osf.io/qzry7/.

\section{Results}

\section{Generation Tasks}

Inclusion generation task. TTo assess awareness, we first compared the percentage of triplets produced to the percentage we would expect by chance $(16.667 \%)$, and then compared performance across groups (see Figure 2). In the inclusion generation task, both the explicit, $M=44.01,95 \%$ CI [37.40, 50.63], $t(92)=8.21, p<.001$, and implicit groups, $M=22.65,95 \%$ CI $[18.79,26.51], t(98)=3.08, p=.003$, produced practice sequence triplets above chance. However, the explicit group produced significantly more triplets than the implicit group, $\Delta M=21.36,95 \%$ CI [13.86, 28.86], $t(190)=5.62, p<.001$. Additionally, both the explicit, $M=7.29,95 \%$ CI [5.57, 9.00], $t(92)=-10.88, p<.001$, and implicit groups, $M=11.39$, 95\% CI [9.79, 12.98], $t(98)=$ -6.56, $p<.001$ produced fewer triplets from the reverse sequence than would be expected by chance. The explicit group however, produced fewer triplets than the implicit group, $\Delta M=-4.10,95 \%$ CI $[-6.42,-1.78], t(190)=-3.48$, $p=.001$.

Exclusion generation task. Turning to the exclusion generation task, participants in the explicit group produced more reverse sequence triplets than would be expected by chance, $M=24.55$, 95\% CI [19.68, 29.42], $t(92)=3.22$, $p=.002$; the implicit group, however, did not, $M=16.41$, $95 \%$ CI $[13.69,19.12], t(98)=-0.19, p=.850$. Furthermore, the explicit group produced more reverse triplets than the implicit group, $\Delta M=8.15,95 \%$ CI $[2.69,13.60]$, $t(190)=2.95, p=.004$. The number of practice sequence triplets however, did not differ from chance for either the explicit, $M=14.80,95 \%$ CI $[11.69,17.91], t(92)=-1.19$, $p=.236$ or implicit groups, $M=15.93,95 \%$ CI [13.39, 18.48], $t(98)=-0.57, p=.569$. Similarly, there was no significant difference between conditions, $\Delta M=-1.14,95 \%$ CI $[-5.10$, 2.83], $t(190)=-0.56, p=.573$.

\section{Serial Reaction Time (SRT) Task}

Prior to all analyses, we removed any participants with accuracy less than $85 \%$ (removing 7 participants) and one 

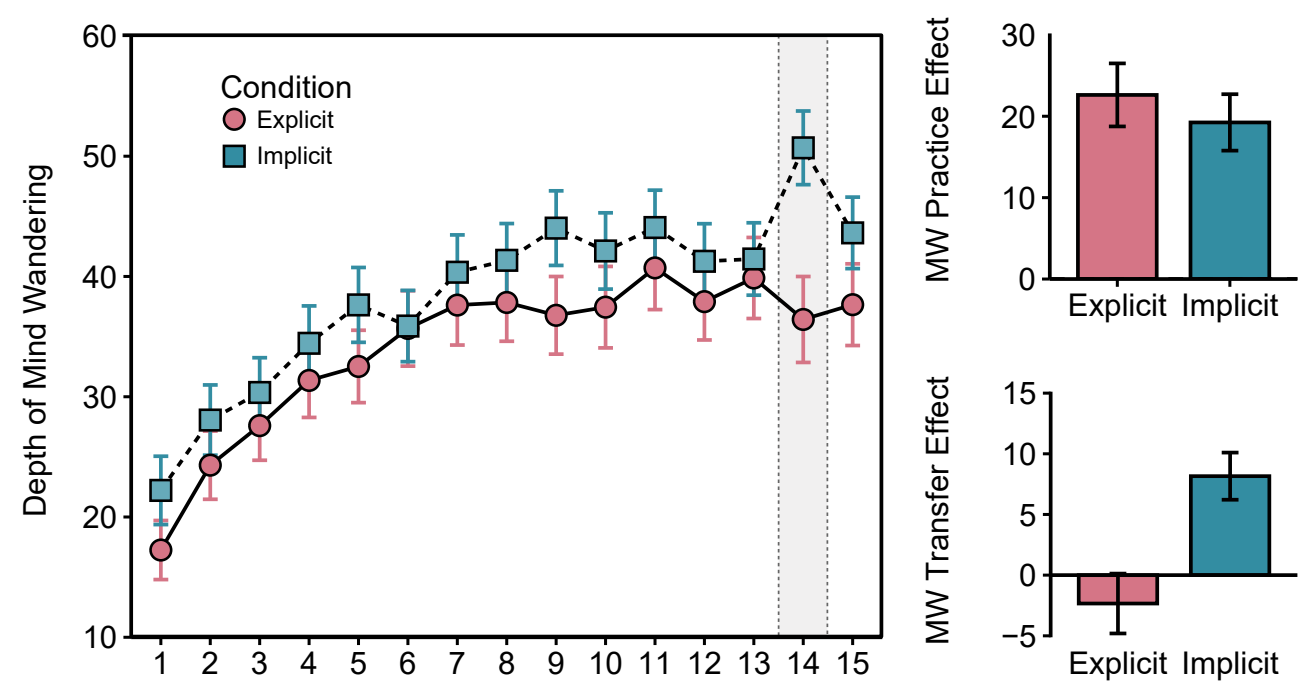

Figure 4. Results from the depth of mind wandering thought probes. Thought probe responses are plotted across trial blocks on the left. Practice effects (reported depth of mind wandering in the 1st block is subtracted from the 13th block) and transfer effects (reported depth of mind wandering in the 14th block is subtracted from the average of the 13th and 15th blocks) are plotted on the right.

participant who did not complete all the trials. Prior to all analyses, we removed error trials, the first trial following a thought probe, removed the first trial of each block, and any responses with reaction times longer than $3000 \mathrm{~ms}$ (removing $1.47 \%$ of observations) before finally applying the non-recursive Van Selst and Jolicoeur outlier removal procedure [Van Selst and Jolicoeur (1994); removing 2.52\% of the remaining observations]. To determine how performance changed across the experiment, we analyzed a "practice effect" and a "transfer effect" for RT and accuracy, separately. The practice effect is the change in performance (RT or accuracy) from the first to the 13th block, and the transfer effect is the change in performance (again, RT or accuracy) from blocks 13 and 15 (averaged) compared to the reversed block, block 14 (see Figure 3 and Appendix A).

We found significant RT practice effects for both the explicit, $M_{d}=-268.74,95 \%$ CI [-307.78, -229.69], $t(86)=$ $-13.68, p<.001, d=-1.43,95 \%$ CI $[-1.72,-1.14]$, and the implicit, $M_{d}=-168.01,95 \%$ CI [-191.09, -144.92], $t(98)=-14.44, p<.001, d=-1.07,95 \%$ CI $[-1.25,-0.89]$. Comparing conditions, we found the RT practice effect for explicit group was significantly larger than the implicit, $\Delta M=-100.73,95 \%$ CI [-144.53, -56.93], $t(184)=-4.54$, $p<.001, d=-0.67,95 \%$ CI [-0.96, -0.37$]$.

Turning to the RT transfer effect, median reaction times were significantly slower in the reversed block (block 14), for the explicit group, $M_{d}=167.55,95 \%$ CI [134.60, 200.49], $t(86)=10.11, p<.001, d=1.19,95 \%$ CI $[0.89,1.5]$, as well as the implicit group, $M_{d}=85.47,95 \%$ CI [72.98, 97.96], $t(98)=13.58, p<.001, d=0.66,95 \%$ CI $[0.55,0.76]$. Com- paring effects across groups, the RT transfer effect was significantly larger for the explicit group, $\Delta M=82.08,95 \% \mathrm{CI}$ $[48.69,115.46], t(184)=4.85, p<.001, d=0.71,95 \% \mathrm{CI}$ $[0.41,1.01]$.

We also analyzed accuracy scores in a similar manner. We found significant accuracy practice effects, in that performance was better in 1st compared to the 13th block, for both the explicit $M_{d}=-0.05,95 \%$ CI [-0.07, -0.03$]$, $t(86)=-4.61, p<.001, d=-0.58,95 \%$ CI $[-0.85,-0.31]$, and implicit groups, $M_{d}=-0.04,95 \%$ CI $[-0.05,-0.02]$, $t(98)=-3.77, p<.001, d=-0.49,95 \%$ CI $[-0.76,-0.22]$. However, this effect did not differ across groups, $\Delta M=0.01$, $95 \%$ CI $[-0.01,0.04], t(184)=0.91, p=.364, d=0.13,95 \%$ CI $[-0.16,0.42]$. More generally, performance was best in the first three blocks, but remained high (88-90\% accurate) throughout the experiment (as in Pasqueli et al. 2019). We also found significant accuracy transfer effects for both the explicit, $M_{d}=-0.05,95 \%$ CI $[-0.07,-0.03], t(86)=-5.21$, $p<.001, d=-0.52,95 \%$ CI $[-0.73,-0.31]$ and implicit, $M_{d}=-0.07,95 \%$ CI $[-0.09,-0.05], t(98)=-7.35, p<.001$, $d=-0.76,95 \%$ CI $[-0.99,-0.53]$ groups showing better accuracy in blocks 13 and 15, compared to block 14, the reverse block; and no significant difference in transfer effects between groups, $\Delta M=0.02,95 \%$ CI $[-0.01,0.04]$, $t(184)=1.07, p=.284, d=0.16,95 \%$ CI $[-0.13,0.45]$.

\section{Thought Probes}

First, we examined whether there were differences in depth of mind wandering between groups. We submitted 
the depth of mind wandering rates to a mixed ANOVA with block as a within-subjects factor (blocks 1 to 13; excluding the final two blocks) and group as between-subjects factor (implicit and explicit). We found a significant main effect of block, $F(6.39,1,175.58)=25.17, M S E=629.93, p<.001$, $\hat{\eta}_{p}^{2}=.120,90 \%$ CI $[0.09,0.15]$, but no main effect of group, $F(1,184)=1.02, M S E=7,499.57, p=.314, \hat{\eta}_{p}^{2}=.006,90 \%$ CI $[0,0.04]$, and no interaction, $F(6.39,1,175.58)=0.42$, $M S E=629.93, p=.879, \hat{\eta}_{p}^{2}=.002,90 \%$ CI $[0,0]$. Put differently, whereas depth of mind wandering increased across training blocks, there was no evidence to suggest that depth of mind wandering differed across groups during the training blocks.

For our primary analyses, we adopted the same analysis plan as above to determine how depth of mind wandering changed throughout the experiment (see Figure 4 and Appendix A). First, we analyzed the practice effect for depth of mind wandering. This mind-wandering practice effect indexes the change in depth of mind wandering that occurred after participants had practiced the SRT sequence. More specifically, it is the reported depth of mind wandering in the 13th block minus the reported depth of mind wandering in the first block.

Here, we found a significant mind-wandering practice effect-an increase in the reported depth of mind wandering from the 1st to the 13th block-for both explicit, $M_{d}=22.62,95 \%$ CI [14.92, 30.32], $t(86)=5.84, p<.001$, $d=0.82,95 \%$ CI $[0.5,1.14]$, and implicit groups, $M_{d}=19.24$, 95\% CI [12.36, 26.12], $t(98)=5.55, p<.001, d=0.66,95 \%$ CI $[0.4,0.92]$; however, no significant difference between groups, $\Delta M=-3.38$, 95\% CI $[-13.60,6.84], t(184)=-0.65$, $p=.515, d=-0.1,95 \%$ CI $[-0.39,0.19]$.

Next, we analyzed the mind-wandering transfer effect across groups. This transfer effect indexes the change in depth of mind wandering that occurred when the sequence was reversed. More specifically, it is the depth of mind wandering reported in the 14 th block (the reversed sequence block) minus the depth of mind wandering reported in the 13th and 15th blocks (the blocks immediately preceding and immediately following the sequence change).

Here, we did not find a significant mind-wandering transfer effect for the explicit group, $M_{d}=-2.34,95 \%$ CI $[-7.24$, 2.57], $t(86)=-0.95, p=.346, d=-0.07,95 \%$ CI $[-0.23$, $0.08]$, but did find a significant mind-wandering transfer effect for the implicit group, $M_{d}=8.16,95 \%$ CI [4.30, 12.01], $t(98)=4.20, p<.001, d=0.28,95 \%$ CI $[0.15,0.41]$. Additionally, the transfer effect was significantly smaller for the explicit versus implicit conditions, $\Delta M=-10.50,95 \%$ CI $[-16.62,-4.37], t(184)=-3.38, p=.001, d=-0.5,95 \%$ CI $[-0.79,-0.2]$. Thus, whereas depth of mind wandering increased in the 14th block for the implicit condition, there was no change in depth of mind wandering across the 13th, 14 th, and 15th blocks in the explicit condition.

\section{Mind wandering, awareness, and SRT performance}

To determine the association between mind wandering, awareness, and SRT performance, we analyzed trial-level reaction times using a linear mixed model with awareness group (explicit versus implicit), depth of mind wandering, and block as fixed effects, and subject as the random effect (see Figure 5). All the blocks were incluedd in the model to estimate both the practice and transfer effects as a function of both awareness and mind wandering. The same outlier removal procedures outlined above were applied here. The full results of this analysis can be found in Appendix B. The mixed model analysis contrasts each level of each factor against a reference level producing multiple interaction terms. Of interest are the three-way interactions between block, mind wandering, and group (all ps < .05; see Appendix B, rows 47 to 60 ), showing that the association between mind wandering and performance differed between the explicit and implicit groups across blocks. Whereas for the explicit group, lower depth of mind wandering was associated with a sharper learning curve, bigger RT practice and transfer effects, for the implicit group, depth of mind wandering was not associated with changes in learning, or with RT practice or RT transfer effects.

To further corroborate this interpretation, we analyzed the data using two linear regression models (see Figure 6), followed-up with Pearson correlations. In the first, we included depth of mind wandering and awareness group (explicit versus implicit) as explanatory variables, and the RT practice effect as the dependent variable. Here, we found a significant interaction between depth of mind wandering and group, $b=2.59$, 95\% CI $[0.75,4.43], t(182)=2.78$, $p=.006$, such that mind wandering was negatively associated with practice effects in the explicit $(r=-.23,95 \% \mathrm{CI}$ $[-.42,-.02], t(85)=-2.22, p=.029)$, but not implicit condition $(r=.15,95 \%$ CI $[-.05, .34], t(97)=1.50, p=.137)$. Interestingly, in the implicit group, although non-significant, the correlation coefficient was in the positive direction.

In the second analysis, we included depth of mind wandering and condition as explanatory variables and the RT transfer effect as the dependent variable. Here again, find a significant interaction between depth of mind wandering and condition, $b=1.89$, 95\% CI [0.51, 3.27], $t(182)=2.71$, $p=.007$. Like the first analysis, we find a negative association between mind wandering and RT transfer effects in the explicit $(r=-.31,95 \%$ CI $[-.49,-.11], t(85)=-3.00$, $p=.004)$, but not implicit condition $(r=-.08,95 \%$ CI $[-.27$, .12 ], $t(97)=-0.79, p=.431)$.

\section{Discussion}

In the present study, we assessed two predictions of the attentional resources account. First, that when task performance and mind wandering do not recruit the same 


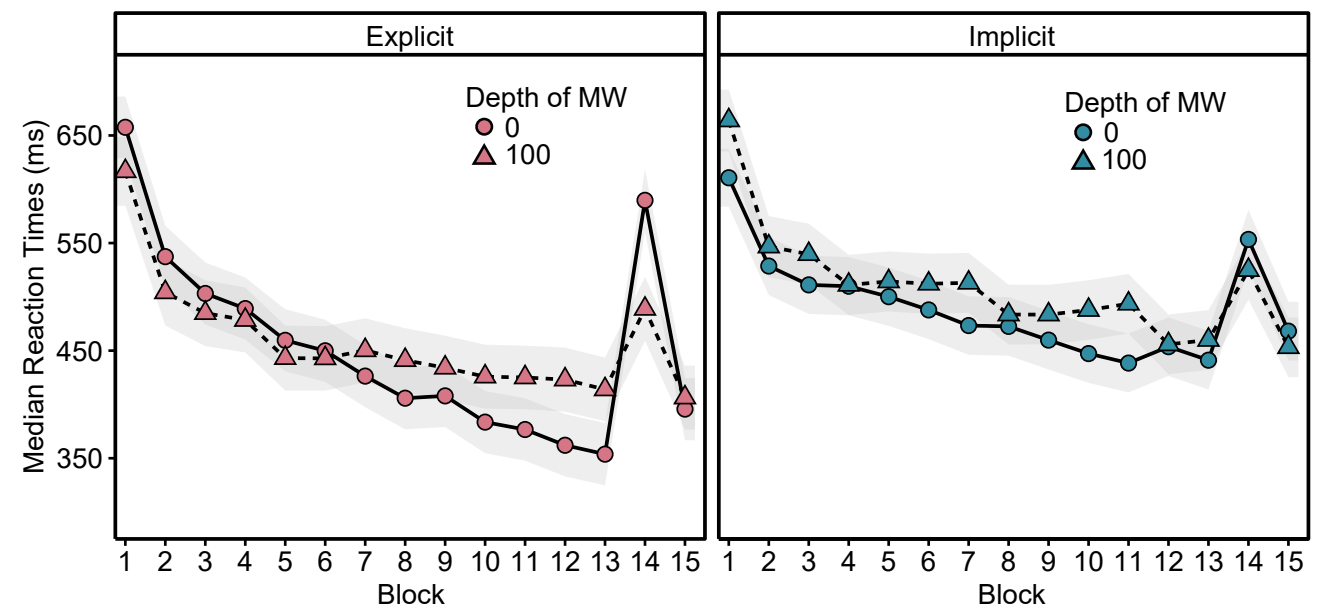

Figure 5. Results from the linear mixed model with depth of mind wandering, block, and awareness as fixed effects and subject as a random effect. Estimated reaction times are plotted for 0 and 100 depth of mind wandering across awareness groups.

attentional resources, mind wandering has no effect on task performance (i.e., mind wandering and task performance can run in parallel without interference). Second, that task-automatization frees up attentional resources that can, in turn, be used for mind wandering (i.e., taskautomatization is positively associated with mind wandering). To these ends, participants completed an SRT task using a 10-element repeating sequence (e.g., Pasquali et al., 2019). One group was made aware of the repeating sequence (i.e., the "explicit" group) and the other was not (i.e., the "implicit" group). Participants practiced the sequence for 13 blocks, at which point they were presented the reverse sequence for one block (block 14) before returning to the training sequence for the final block (block 15). After each block of trials, we presented participants a thought probe to gauge their depth of mind wandering and, at the end of the experiment, we assessed their awareness of the repeating sequence using two sequence generation tasks.

To assess awareness of the repeating sequence, we included two sequence generation tasks. We found that both groups could produce training sequence triplets and refrain from producing reverse sequence triplets. However, the explicit group outperformed the implicit group in both cases and only participants in the explicit group could produce the reverse sequence triplets above chance. Taken together, these results suggest that the knowledge acquired in the explicit learning group was indeed explicit, as they were better able to mentally reconstruct the training sequence and even reverse it. Importantly, these results also suggest that knowledge acquired in the implicit learning group was likely implicit, as participants in this group could not reproduce the reverse sequence at rates above chance. Critically, these results indicate that our study was able to effectively assess the association between mind wandering and task performance when varying the resources necessary to maintain adequate task performance. This, in turn, allowed us to test two key predictions of the attentional resource account: that (a) a focal task that does not draw on the same attentional resources as mind wandering can run in parallel with mind wandering without interference, and (b) task-automatization is associated with increases in mind wandering.

To test the first prediction (that task-automatization is associated with increases in mind wandering), we examined changes in mind wandering and task performance before and after participants practiced the sequence. Here, we found that SRT reaction time task performance improved considerably with increased time on task (indicating that task performance became increasingly automated), while at the same time, depth of mind wandering increased. These findings provide novel evidence in favor of the taskautomatization prediction of the attentional resources account. However, it is important to note that we still cannot rule out other possible (non-mutually-exclusive) casual factors such as boredom, fatigue, motivation (e.g., Brosowsky et al., in press-a), or waning cognitive control (e.g., Thomson, Besner, \& Smilek, 2015). Future work is needed to tease apart the relative contributions of these factors and develop a more comprehensive understanding of why people gradually disengage from their primary task. Our results, however, suggest that task automatization is a viable factor that needs to be considered.

To test the second prediction (that a focal task can run in parallel with mind wandering without interference), we examined the association between mind wandering, learning, and awareness. In the explicit learning group, we found that depth of mind wandering was negatively associated with learning rates (as indexed by both practice and transfer 

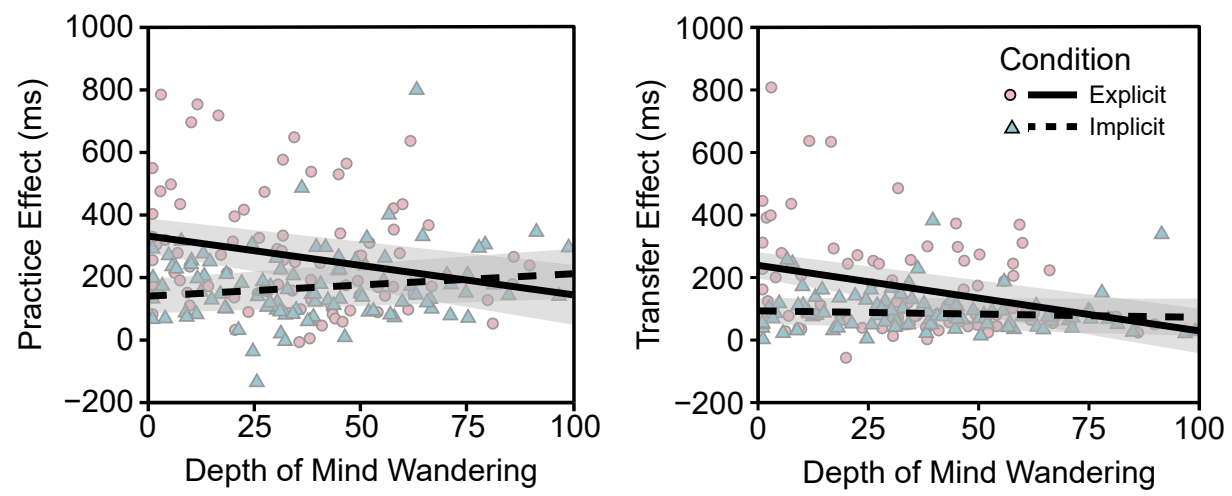

Figure 6. Results from the linear regression models plotted over participant data. Practice effects are plotted against depth of mind wandering (left) and transfer effects are plotted against depth of mind wandering (right).

effects). However, this was not the case for the implicit group, whose learning rates were not associated with their reported depth of mind wandering. This result likewise provides novel evidence for the attentional resources account: Explicit learning requires attentional resources and is therefore disrupted when participants engaged in deeper bouts of mind wandering. In contrast, implicit learning does not require attentional resources and is therefore unaffected by a participants' depth of mind wandering. ${ }^{3}$

In addition to the foregoing, our results provide novel evidence to suggest that mind wandering (defined as taskunrelated thought) may not compete for attentional resources during implicit learning in an SRT task. The relation between attention and implicit learning has been, and is still, a heavily debated topic (Cleeremans et al., 2019; Jiménez, 2003). Typically, dual-task paradigms are used to determine whether implicit learning is impaired under attentional load. Although many studies have demonstrated unimpaired learning under attentional load (e.g., Cohen et al., 1990; Frensch \& Miner, 1995; Reed \& Johnson, 1994; Shanks \& Johnstone, 1999), others have shown impaired learning, slowed reaction times, and diminished transfer effects (e.g., Jimenez \& Vazquez, 2005; Shanks \& Channon, 2002; Shanks, Rowland, \& Ranger, 2005). Here, however, we find self-reports of inattention (i.e., mind wandering) were not associated with performance in the implicit group, but that they were negatively associated with performance in the explicit group. These results provide strong support for the view that implicit learning, unlike explicit learning, is unimpaired under attentional load using a novel measure of inattention. This is an important contribution to the ongoing debate about the relation of attention and implicit learning (Cleeremans et al., 2019; Jiménez, 2003).

A secondary aim of the current study was to further validate the use of SRT paradigms to study implicit learning in an online setting (Sævland \& Norman, 2016). To that end, we closely modelled our methodology after Pasquali et al. (2019). As predicted, we found robust reaction-time practice and transfer effects in both the explicit and implicit learning conditions, and differences in learning performance across learning conditions (e.g. Cleeremans et al., 1998; Cohen et al., 1990; Nissen \& Bullemer, 1987; Reed \& Johnson, 1994; Shanks \& Johnstone, 1999; Stadler, 1993). We also observed the expected dissociations between learning conditions using the generation tasks (e.g., Destrebecqz \& Cleeremans, 2001; Pasquali et al., 2019). In our study, however, both groups produced training sequence triplets at the same rate during the exclusion generation task (both were at chance). This differed from Pasquali et al., who had found that participants in the implicit condition were above chance (which indicated that these participants failed to explicitly exclude the training sequence). Whether this difference occurred because of the online procedure, fewer training blocks, the change in sequence length, or a Type 2 error is unclear. Despite this difference, however, we provide another successful replication (Sævland \& Norman, 2016) of the classic explicit and implicit learning effects that one would expect to find the in the laboratory. Our results are therefore heartening as they provide further evidence to suggest the feasibility of using online procedures to measure incidental sequence learning (see osf.io/qzry7/ for experiment procedures and code).

When employing online procedures, there is always an inherent loss of experimental control over how stimuli are presented and how participants engage with the task (e.g. Armitage \& Eerola, 2020; Buhrmester et al., 2018; Casler et al., 2013; Dandurand et al., 2008; Hilbig, 2016; Leeuw \&

\footnotetext{
${ }^{3}$ It should be noted that our results are generally inconsistent with the work by Franklin et al. (2016), who found that implicit sequence learning was negatively associated with mind wandering. As mentioned in our Introduction, however, there were numerous methodological and statistical shortcomings with Franklin et al.'s study that suggest that their results should be interpreted with caution. Given that the present study addressed all of these concerns, we think it provides a more appropriate test of the effect of mind wandering on implicit and explicit learning.
} 
Motz, 2016). In the Introduction, we briefly discussed measures we took to mitigate potential problems associated with conducting our study online. There are, however, a few notable limitations worth discussing further. ${ }^{4}$ First, in our study, we did not give participants explicit instructions on how they should complete the task. We did not, for instance, instruct them about which fingers should be used for which response keys or give any explicit instruction about response strategies. Of course, in an online setting, we are unable to monitor how participants engage in the task or whether they change their response strategies during the task. We therefore modelled our design after Pasquali et al. (2019) who likewise did not present such instructions. However, in hindsight, this could have been problematic because we adopted the five-choice SRT design (as described by Pasquali et al., 2019; and used elsewhere, Schmitz, Pasquali, Cleeremans, \& Peigneux, 2013) and it is not immediately obvious how participants coped with the five response keys (e.g., we do not know which finger was used for the middle response, nor do we know whether they changed their response strategy at some point in the experiment). In any case, our ability to test the specific hypotheses outlined here were not dependent upon the provision of such instructions and, furthermore, we successfully replicated classic explicit/implicit sequence learning effects (e.g., Cleeremans, 1993; Cleeremans, Destrebecqz, \& Boyer, 1998; Cohen, Ivry, \& Keele, 1990; Nissen \& Bullemer, 1987; Reed \& Johnson, 1994; Shanks \& Johnstone, 1999; Stadler, 1993) by adapting the methodology outlined by Pasquali et al. (2019; some differences in design are noted in the Introduction). We therefore do not think this instructional omission meaningfully bears on the results of our study, but we do suggest that future work using the SRT paradigm in an online setting might consider whether such limitations are important for their use-cases (e.g., Jiménez et al., 2008).

Finally, we turn to a surprising, but potentially important, result: Participants in the implicit group reported a considerable increase in mind-wandering depth during the reversed-sequence block. This contrasts sharply with the explicit group, who showed no differences in their mindwandering depth across the three final blocks. The question, then, is what explains this difference in mind-wandering reports between the implicit and explicit groups?

Perhaps the simplest explanation is that the change in task performance caused an increase in mind wandering in the implicit, but not the explicit, group. Recall that participants in the implicit group were unaware that there was a repeating sequence throughout most of the SRT task. Thus, the sudden and inexplicable change in performance occurring when the task switched from the repeated sequence to the novel, reverse sequence (i.e., block 14) may have caused participants in the implicit group to experience more taskrelated interferences (e.g., "Why am I suddenly doing so poorly?"), which may have been reported as increased mind wandering. In contrast, participants in the explicit condition, who were aware of the repeating sequence, were likely less confused about the sudden shift in their performance during block 14; indeed, participants in this group ought to have immediately realized that the sequence was switched, which would result in fewer task-related interferences (and hence, no increase in reported depth of mind wandering) because these participants would have been fully aware of why their performance was temporarily poorer.

A second potential explanation is that participants were inferring their depth of mind wandering based on their performance (Head \& Helton, 2018). However, it is unlikely that participants were using a simple heuristic that poor performance signals deeper mind wandering. Indeed, here we observed concurrent increases in performance and depth of mind wandering throughout the task. Furthermore, participants in the explicit group showed a larger performance drop than the implicit group, yet they did not report changes in their depth of mind wandering. While our results indicate that it is unlikely that participants infer their depth of mind wandering based on performance, per se, it is plausible that they instead infer mind wandering based on the discrepancy between their performance and their normative expectations about their performance (Whittlesea, 2002; Whittlesea \& Williams, 2000, 2001a, 2001b). That is, when people perform worse than they expect in a given context, they might infer that they were inattentive or mind wandering. Participants in the implicit group were unaware that the task contained a repeated sequence and, consequently, would have been unable to accurately attribute their poor performance to a change in the task. This would result in a large discrepancy between their performance and their expected performance. In contrast, participants who were aware that there was a repeating sequence (i.e., those in the explicit group) were likely also aware that the sequence changed. Therefore, they could accurately attribute their poor performance to a change in the task, which would preclude the discrepancy experienced by participants in the implicit group. This possibility is, of course, speculative, but it would suggest that awareness of task structure and performance may influence self-reports of mind wandering and could potentially have important implications for mindwandering research. Moving forward, more research on this topic is needed.

\section{Concluding Remarks}

Collectively, these findings demonstrate that the costs of mind wandering critically depend on the resource requirements of the primary task. When task requirements are high (e.g. during explicit learning), mind wandering is associated

\footnotetext{
${ }^{4}$ We thank Dr. Jiménez for raising these issues.
} 
with considerable disruption. However, when task demands are low (e.g. during implicit learning), mind wandering can go on in parallel and even serve as a marker of successful learning. These findings support several key predictions of the attentional resource theory of mind wandering (Smallwood \& Schooler, 2006), while also illuminating the circumstances under which mind wandering is most versus least likely to be problematic.

\section{References}

Antrobus, J. S. (1968). Information theory and stimulusindependent thought. British Journal of Psychology, 59(4), 423-430.

Armitage, J., \& Eerola, T. (2020). Reaction time data in music cognition: Comparison of pilot data from lab, crowdsourced, and convenience Web samples. Frontiers in Psychology, 10, 2883.

Aust, F., \& Barth, M. (2018). papaja: Create APA manuscripts with R Markdown. Retrieved from https://github. $\mathrm{com} / \mathrm{crsh} / \mathrm{papaja}$

Baker, C. I., Olson, C. R., \& Behrmann, M. (2004). Role of attention and perceptual grouping in visual statistical learning. Psychological Science, 15(7), 460-466.

Bates, D., Mächler, M., Bolker, B., \& Walker, S. (2015). Fitting linear mixed-effects models using lme4. Journal of Statistical Software, 67(1), 1-48. https://doi.org/ 10.18637/jss.v067.i01

Behmer Jr, L. P., \& Crump, M. J. (2016). Crunching Big Data with Fingertips: How Typists Tune Their Performance Toward the Statistics of Natural Language. In Big data in cognitive science (pp. 329-345). Psychology Press.

Bejjani, C., Tan, S., \& Egner, T. (2020). Performance feedback promotes proactive but not reactive adaptation of conflict-control. Journal of Experimental Psychology: Human Perception and Performance, 46(4), 369.

Brosowsky, N. P., \& Crump, M. J. C. (2018). Memory-guided selective attention: Single experiences with conflict have long-lasting effects on cognitive control. Journal of Experimental Psychology: General, 147, 1134-1153. https://doi.org/10.1037/xge0000431

Brosowsky, N. P., \& Crump, M. J. C. (2020). Contextual recruitment of selective attention can be updated via changes in task relevance. Canadian Journal of Experimental Psychology/Revue Canadienne de Psychologie Expérimentale, Advance online publication. https://doi.org/https://doi.org/10.1037/ cep0000221

Brosowsky, N. P., Degutis, J., Esterman, M., Smilek, D., \& Seli, P. (in press-a). Mind wandering, motivation, and task performance over time. Psychology of Consciousness: Theory Research, and Practice.
Brosowsky, N. P., Murray, Samuel, Schooler, Jonathan, \& Seli, P. (under review-b). Thought dynamics under task demands: The influence of task difficulty on thought constraint.

Buhrmester, M. D., Talaifar, S., \& Gosling, S. D. (2018). An evaluation of Amazon's Mechanical Turk, its rapid rise, and its effective use. Perspectives on Psychological Science, 13(2), 149-154.

Casler, K., Bickel, L., \& Hackett, E. (2013). Separate but equal? A comparison of participants and data gathered via Amazon's MTurk, social media, and faceto-face behavioral testing. Computers in Human Behavior, 29(6), 2156-2160.

Chmielewski, M., \& Kucker, S. C. (2020). An MTurk crisis? Shifts in data quality and the impact on study results. Social Psychological and Personality Science, 11(4), 464-473.

Cleeremans, A., Allakhverdov, V., \& Kuvaldina, M. (2019). Implicit learning: 50 Years on. Routledge.

Cleeremans, A., Destrebecqz, A., \& Boyer, M. (1998). Implicit learning: News from the front. Trends in Cognitive Sciences, 2(10), 406-416.

Cohen, A., Ivry, R. I., \& Keele, S. W. (1990). Attention and structure in sequence learning. Journal of Experimental Psychology: Learning, Memory, and Cognition, 16(1), 17.

Cowan, N. (1988). Evolving conceptions of memory storage, selective attention, and their mutual constraints within the human information-processing system. Psychological Bulletin, 104(2), 163.

Crump, M. J., Brosowsky, N. P., \& Milliken, B. (2017). Reproducing the location-based context-specific proportion congruent effect for frequency unbiased items: A reply to Hutcheon and Spieler (2016). Quarterly Journal of Experimental Psychology, 70(9), 17921807.

Crump, M. J., Lai, W., \& Brosowsky, N. P. (2019). Instance theory predicts information theory: Episodic uncertainty as a determinant of keystroke dynamics. Canadian Journal of Experimental Psychology/Revue Canadienne de Psychologie Expérimentale, 73(4), 203.

Cunningham, S., Scerbo, M. W., \& Freeman, F. G. (2000). The electrocortical correlates of daydreaming during vigilance tasks. Journal of Mental Imagery.

Dale, R., Duran, N. D., \& Morehead, J. R. (2012). Prediction during statistical learning, and implications for the implicit/explicit divide. Advances in Cognitive Psychology, 8(2), 196.

Dandurand, F., Shultz, T. R., \& Onishi, K. H. (2008). Comparing online and lab methods in a problem-solving experiment. Behavior Research Methods, 40(2), 428-434. 
Destrebecqz, A., \& Cleeremans, A. (2001). Can sequence learning be implicit? New evidence with the process dissociation procedure. Psychonomic Bulletin \& Review, 8(2), 343-350.

Fox, J., \& Weisberg, S. (2018). Visualizing fit and lack of fit in complex regression models with predictor effect plots and partial residuals. Journal of Statistical Software, 87(9), 1-27. https://doi.org/10.18637/jss. v087.i09

Fox, J., \& Weisberg, S. (2019). An R companion to applied regression (Third). Thousand Oaks CA: Sage. Retrieved from https://socialsciences.mcmaster.ca/ jfox/Books/Companion/

Franklin, M. S., Smallwood, J., Zedelius, C. M., Broadway, J. M., \& Schooler, J. W. (2016). Unaware yet reliant on attention: Experience sampling reveals that mind-wandering impedes implicit learning. Psychonomic Bulletin \& Review, 23(1), 223-229.

Frensch, P. A., \& Miner, C. S. (1995). Zur Rolle des Arbeitsgedächtnisses beim impliziten Sequenzlernen. [The role of working memory in implicit sequence learning.]. Zeitschrift Für Experimentelle Psychologie, 42(4), 545-575.

Giambra, L. M. (1989). Task-unrelated thought frequency as a function of age: A laboratory study. Psychology and Aging, 4(2), 136.

Gil-Jardiné, C., Née, M., Lagarde, E., Schooler, J., Contrand, B., Orriols, L., \& Galera, C. (2017). The distracted mind on the wheel: Overall propensity to mind wandering is associated with road crash responsibility. PloS One, 12(8).

Hasenkamp, W. (2013). How to focus a wandering mind. Greater Good Magazine.

Head, J., \& Helton, W. S. (2018). The troubling science of neurophenomenology. Experimental Brain Research, 236(9), 2463-2467.

Hilbig, B. E. (2016). Reaction time effects in lab-versus Webbased research: Experimental evidence. Behavior Research Methods, 48(4), 1718-1724.

Jiang, Y., \& Chun, M. M. (2001). Selective attention modulates implicit learning. The Quarterly Journal of Experimental Psychology: Section A, 54(4), 11051124.

Jimenez, L., \& Vazquez, G. A. (2005). Sequence learning under dual-task conditions: Alternatives to a resource-based account. Psychological Research, 69(5-6), 352-368.

Jiménez, L. (2003). Attention and implicit learning (Vol. 48). John Benjamins Publishing.

Jiménez, L. (2008). Taking patterns for chunks: Is there any evidence of chunk learning in continuous serial reaction-time tasks? Psychological Research, 72(4), 387-396.
Jiménez, L., \& Mendez, C. (1999). Which attention is needed for implicit sequence learning? Journal of Experimental Psychology: Learning, Memory, and Cognition, 25(1), 236.

Jiménez, L., Méndez, A., Pasquali, A., Abrahamse, E., \& Verwey, W. (2011). Chunking by colors: Assessing discrete learning in a continuous serial reaction-time task. Acta Psychologica, 137(3), 318-329.

Johnson, M. K., \& Hirst, W. (1993). MEM: Memory subsystems as processes. Theories of Memory, 1, 241-286.

Kassambara, A. (2019). Ggpubr: 'Ggplot2' based publication ready plots. Retrieved from https://CRAN.Rproject.org/package=ggpubr

Keele, S. W., Ivry, R., Mayr, U., Hazeltine, E., \& Heuer, H. (2003). The cognitive and neural architecture of sequence representation. Psychological Review, $110(2), 316$.

Keele, S. W., Jennings, P., Jones, S., Caulton, D., \& Cohen, A. (1995). On the modularity of sequence representation. Journal of Motor Behavior, 27(1), 17-30.

Kim, J., Gabriel, U., \& Gygax, P. (2019). Testing the effectiveness of the Internet-based instrument PsyToolkit: A comparison between web-based (PsyToolkit) and lab-based (E-Prime 3.0) measurements of response choice and response time in a complex psycholinguistic task. PloS One, 14(9), e0221802.

Krimsky, M., Forster, D. E., Llabre, M. M., \& Jha, A. P. (2017). The influence of time on task on mind wandering and visual working memory. Cognition, 169, 84-90.

Kuznetsova, A., Brockhoff, P. B., \& Christensen, R. H. B. (2017). lmerTest package: Tests in linear mixed effects models. Journal of Statistical Software, 82(13), 1-26. https://doi.org/10.18637/jss.v082.i13

Leeuw, J. R. de, \& Motz, B. A. (2016). Psychophysics in a Web browser? Comparing response times collected with JavaScript and Psychophysics Toolbox in a visual search task. Behavior Research Methods, 48(1), 112.

Logan, G. D. (1988). Toward an instance theory of automatization. Psychological Review, 95, 492-527. https://doi.org/10.1037/0033-295X.95.4.492

Logan, G. D., \& Compton, B. J. (1998). Attention and automaticity. Visual Attention, 8, 108-131.

McVay, J. C., \& Kane, M. J. (2010). Does mind wandering reflect executive function or executive failure? Comment on Smallwood and Schooler (2006) and Watkins (2008). Psychological Bulletin, 136(2), 188197. https://doi.org/10.1037/a0018298

McVay, J. C., \& Kane, M. J. (2012). Drifting from slow to "d'oh!": Working memory capacity and mind wandering predict extreme reaction times and executive control errors. Journal of Experimental Psychology: Learning, Memory, and Cognition, 38(3), 
525.

Metcalfe, J., \& Xu, J. (2016). People mind wander more during massed than spaced inductive learning. Journal of Experimental Psychology: Learning, Memory, and Cognition, 42(6), 978.

Mooneyham, B. W., \& Schooler, J. W. (2013). The costs and benefits of mind-wandering: A review. Canadian Journal of Experimental Psychology/Revue Canadienne de Psychologie Expérimentale, 67(1), 11.

Mooneyham, B. W., \& Schooler, J. W. (2016). Mind wandering minimizes mind numbing: Reducing semanticsatiation effects through absorptive lapses of attention. Psychonomic Bulletin \& Review, 23(4), 12731279. https://doi.org/10.3758/s13423-015-0993-2

Moors, A., \& De Houwer, J. (2006). Automaticity: A theoretical and conceptual analysis. Psychological Bulletin, 132(2), 297-326. https://doi.org/10.1037/00332909.132.2.297

Newell, A., \& Rosenbloom, P. S. (1981). Mechanisms of skill acquisition and the law of practice. In Cognitive Skills and their Acquisition (pp. 1-55). Retrieved from http://books.google.com/books?hl=en\& $\mathrm{lr}=\& i d=7 \mathrm{oEtr} 1 \mathrm{KvMbgC} \& \mathrm{oi}=\mathrm{fnd} \& \mathrm{pg}=\mathrm{PA} 1 \& \mathrm{dq}=$ newell+rosenbloom $+1981 \&$ ots $=1$ Kc_ugE10m\& sig=x567YdNZZZGs1QEjLXSLgU2hQvo

Nissen, M. J., \& Bullemer, P. (1987). Attentional requirements of learning: Evidence from performance measures. Cognitive Psychology, 19(1), 1-32.

Pasquali, A., Cleeremans, A., \& Gaillard, V. (2019). Reversible second-order conditional sequences in incidental sequence learning tasks. Quarterly Journal of Experimental Psychology, 72(5), 1164-1175. https://doi.org/10.1177/1747021818780690

R Core Team. (2019). R: A language and environment for statistical computing. Vienna, Austria: R Foundation for Statistical Computing. Retrieved from https://www.R-project.org/

Reber, A. S. (1989). Implicit learning and tacit knowledge. Journal of Experimental Psychology: General, 118(3), 219.

Reed, J., \& Johnson, P. (1994). Assessing implicit learning with indirect tests: Determining what is learned about sequence structure. Journal of Experimental Psychology: Learning, Memory, and Cognition, 20(3), 585.

Reichle, E. D., Reineberg, A. E., \& Schooler, J. W. (2010). Eye movements during mindless reading. Psychological Science, 21(9), 1300-1310.

Risko, E. F., Anderson, N., Sarwal, A., Engelhardt, M., \& Kingstone, A. (2012). Everyday Attention: Variation in Mind Wandering and Memory in a Lecture. Applied Cognitive Psychology, 26(2), 234-242. https://doi.org/10.1002/acp.1814
Schneider, W., \& Shiffrin, R. M. (1977). Controlled and automatic human information processing: I. Detection, search, and attention. Psychological Review, 84(1), 1.

Schumacher, E. H., \& Schwarb, H. (2009). Parallel response selection disrupts sequence learning under dualtask conditions. Journal of Experimental Psychology: General, 138(2), 270.

Schwarb, H., \& Schumacher, E. H. (2012). Generalized lessons about sequence learning from the study of the serial reaction time task. Advances in Cognitive Psychology, 8(2), 165.

Seger, C. A. (1994). Implicit learning. Psychological Bulletin, 115(2), 163.

Seli, P., Cheyne, J. A., \& Smilek, D. (2013). Wandering minds and wavering rhythms: Linking mind wandering and behavioral variability. Journal of Experimental Psychology: Human Perception and Performance, 39(1), 1.

Seli, P., Kane, M. J., Smallwood, J., Schacter, D. L., Maillet, D., Schooler, J. W., \& Smilek, D. (2018). Mind-wandering as a natural kind: A familyresemblances view. Trends in Cognitive Sciences, 22(6), 479-490.

Seli, P., Konishi, M., Risko, E. F., \& Smilek, D. (2018). The role of task difficulty in theoretical accounts of mind wandering. Consciousness and Cognition, 65, 255262.

Seli, P., Risko, E. F., Smilek, D., \& Schacter, D. L. (2016). Mindwandering with and without intention. Trends in Cognitive Sciences, 20(8), 605-617.

Shanks, D. R., \& Channon, S. (2002). Effects of a secondary task on" implicit" sequence learning: Learning or performance? Psychological Research, 66(2), 99109.

Shanks, D. R., \& Johnstone, T. (1999). Evaluating the relationship between explicit and implicit knowledge in a sequential reaction time task. Journal of Experimental Psychology: Learning, Memory, and Cognition, 25(6), 1435.

Shanks, D. R., Rowland, L. A., \& Ranger, M. S. (2005). Attentional load and implicit sequence learning. Psychological Research, 69(5-6), 369-382.

Shiffrin, R. M., \& Schneider, W. (1977). Controlled and automatic human information processing: II. Perceptual learning, automatic attending and a general theory. Psychological Review, 84, 127-190. https://doi.org/10.1037/0033-295X.84.2.127

Singmann, H., Bolker, B., Westfall, J., Aust, F., \& Ben-Shachar, M. S. (2019). Afex: Analysis of factorial experiments. Retrieved from https://CRAN.R-project. org/package $=$ afex

Smallwood, J. (2013). Distinguishing how from why the 
mind wanders: A process-occurrence framework for self-generated mental activity. Psychological Bulletin, 139(3), 519.

Smallwood, J., Beach, E., Schooler, J. W., \& Handy, T. C. (2008a). Going AWOL in the brain: Mind wandering reduces cortical analysis of external events. Journal of Cognitive Neuroscience, 20(3), 458-469.

Smallwood, J., Brown, K. S., Tipper, C., Giesbrecht, B., Franklin, M. S., Mrazek, M. D., ... Schooler, J. W. (2011). Pupillometric evidence for the decoupling of attention from perceptual input during offline thought. PloS One, 6(3).

Smallwood, J., Davies, J. B., Heim, D., Finnigan, F., Sudberry, M., O’Connor, R., \& Obonsawin, M. (2004). Subjective experience and the attentional lapse: Task engagement and disengagement during sustained attention. Consciousness and Cognition, 13(4), 657690.

Smallwood, J. M., Baracaia, S. F., Lowe, M., \& Obonsawin, M. (2003). Task unrelated thought whilst encoding information. Consciousness and Cognition, 12(3), 452-484.

Smallwood, J., McSpadden, M., \& Schooler, J. W. (2008b). When attention matters: The curious incident of the wandering mind. Memory \& Cognition, 36(6), 1144-1150.

Smallwood, J., \& Schooler, J. W. (2006). The restless mind. Psychological Bulletin, 132(6), 946.

Smallwood, J., \& Schooler, J. W. (2015). The science of mind wandering: Empirically navigating the stream of consciousness. Annual Review of Psychology, 66, 487-518.

Smith, A. P., Brosowsky, N. P., Daniel, R., Meier, M. E., \& Seli, P. (under review). Fixation, flexibility, and creativity: The dynamics of mind wandering.

Stadler, M. A. (1993). Implicit serial learning: Questions inspired by Hebb (1961). Memory \& Cognition, 21(6), 819-827.

Sævland, W., \& Norman, E. (2016). Studying different tasks of implicit learning across multiple test sessions conducted on the web. Frontiers in Psychology, 7, 808.

Teasdale, J. D., Dritschel, B. H., Taylor, M. J., Proctor, L., Lloyd, C. A., Nimmo-Smith, I., \& Baddeley, A. D. (1995). Stimulus-independent thought depends on central executive resources. Memory \& Cognition, 23(5), 551-559. https://doi.org/10.3758/ BF03197257

Thomson, D. R., Besner, D., \& Smilek, D. (2013). In pursuit of off-task thought: Mind wandering-performance trade-offs while reading aloud and color naming. Frontiers in Psychology, 4, 360.

Thomson, D. R., Besner, D., \& Smilek, D. (2015). A resource- control account of sustained attention: Evidence from mind-wandering and vigilance paradigms. Perspectives on Psychological Science, 10(1), 82-96.

Thomson, D. R., Seli, P., Besner, D., \& Smilek, D. (2014). On the link between mind wandering and task performance over time. Consciousness and Cognition, 27, 14-26. https://doi.org/10.1016/j.concog.2014.04. 001

Turk-Browne, N. B., Jungé, J. A., \& Scholl, B. J. (2005). The automaticity of visual statistical learning. Journal of Experimental Psychology: General, 134(4), 552.

Unsworth, N., \& Engle, R. W. (2005). Individual differences in working memory capacity and learning: Evidence from the serial reaction time task. Memory \& Cognition, 33(2), 213-220.

Van Selst, M., \& Jolicoeur, P. (1994). A solution to the effect of sample size on outlier elimination. The Quarterly Journal of Experimental Psychology Section A, 47, 631-650. https://doi.org/10.1080/ 14640749408401131

Whittlesea, B. W. A. (2002). False memory and the discrepancy-attribution hypothesis: The prototype-familiarity illusion. Journal of Experimental Psychology: General, 131(1), 96-115. https: //doi.org/10.1037/0096-3445.131.1.96

Whittlesea, B. W. A., \& Williams, L. D. (2000). The source of feelings of familiarity: The discrepancy-attribution hypothesis. Journal of Experimental Psychology: Learning, Memory, and Cognition, 26(3), 547-565. https://doi.org/10.1037/0278-7393.26.3.547

Whittlesea, B. W. A., \& Williams, L. D. (2001a). The discrepancy-attribution hypothesis: II Expectation, uncertainty, surprise, and feelings of familiarity. Journal of Experimental Psychology: Learning, Memory, and Cognition, 27(1), 14-33. https: //doi.org/10.1037/0278-7393.27.1.14

Whittlesea, B. W. A., \& Williams, L. D. (2001b). The discrepancy-attribution hypothesis: I The heuristic basis of feelings and familiarity. Journal of Experimental Psychology: Learning, Memory, and Cognition, 27(1), 3-13. https://doi.org/10.1037/02787393.27.1.3

Wickham, H. (2016). Ggplot2: Elegant graphics for data analysis. Springer-Verlag New York. Retrieved from https://ggplot2.tidyverse.org

Wickham, H., François, R., Henry, L., \& Müller, K. (2019). Dplyr: A grammar of data manipulation. Retrieved from https://CRAN.R-project.org/package=dplyr

Wickham, H., \& Henry, L. (2019). Tidyr: Tidy messy data. Retrieved from https://CRAN.R-project.org/ package $=$ tidyr

Wilke, C. O. (2019). Cowplot: Streamlined Plot Theme and Plot Annotations for 'ggplot2'. Retrieved from 
https: / /CRAN.R-project.org/package=cowplot

Willingham, D. B., Nissen, M. J., \& Bullemer, P. (1989). On the development of procedural knowledge. Journal of Experimental Psychology: Learning, Memory, and Cognition, 15(6), 1047.

Xu, J., \& Metcalfe, J. (2016). Studying in the region of proximal learning reduces mind wandering. Memory \& Cognition, 44(5), 681-695.

Yanko, M. R., \& Spalek, T. M. (2014). Driving with the wandering mind: The effect that mind-wandering has on driving performance. Human Factors, 56(2), 260-269. 
Table Al

\section{Appendix A}

Mean and standard errors for median reaction times (RT), error rates (\%) and depth of mind wandering as a function of block awareness conditions

\begin{tabular}{rlllllll}
\hline & \multicolumn{3}{c}{ Explicit } & & \multicolumn{3}{l}{ Implicit } \\
\cline { 2 - 3 } \cline { 6 - 7 } Block & RT & Error & MW & & RT & Error & MW \\
\hline 1 & $625.17(22.61)$ & $7.13(0.75)$ & $17.25(2.46)$ & & $600.82(16.78)$ & $7.81(0.63)$ & $22.21(2.84)$ \\
2 & $514.29(18.09)$ & $6.96(0.62)$ & $24.31(2.84)$ & & $520.99(14.01)$ & $6.95(0.57)$ & $28.06(2.92)$ \\
3 & $480.62(18.93)$ & $7.43(0.71)$ & $27.6(2.88)$ & & $502.98(13.55)$ & $7.01(0.62)$ & $30.35(2.89)$ \\
4 & $466.58(18.56)$ & $8.87(0.95)$ & $31.36(3.08)$ & & $496.28(13.82)$ & $8.88(0.79)$ & $34.44(3.11)$ \\
5 & $441.32(16.89)$ & $9.6(0.87)$ & $32.52(3.01)$ & & $490.49(14.06)$ & $9.53(0.65)$ & $37.64(3.11)$ \\
6 & $429.33(16.79)$ & $10.03(0.87)$ & $35.68(3.13)$ & & $484.96(13.31)$ & $9.98(0.74)$ & $35.88(2.96)$ \\
7 & $419.59(16.83)$ & $9.77(0.71)$ & $37.62(3.32)$ & & $473.86(13.24)$ & $9.96(0.72)$ & $40.35(3.1)$ \\
8 & $398.85(15.55)$ & $10.8(0.87)$ & $37.84(3.22)$ & & $464.81(12.89)$ & $10.92(0.85)$ & $41.34(3.06)$ \\
9 & $391.19(16.53)$ & $9.85(0.9)$ & $36.77(3.23)$ & & $453.06(13.34)$ & $11.03(0.89)$ & $44.02(3.1)$ \\
10 & $377.09(16.55)$ & $11.87(0.87)$ & $37.45(3.39)$ & & $449.89(13.76)$ & $10.64(0.72)$ & $42.12(3.17)$ \\
11 & $369.24(16.58)$ & $11.8(0.97)$ & $40.71(3.47)$ & & $446.57(13.3)$ & $10.94(0.84)$ & $44.07(3.1)$ \\
12 & $360.83(16.08)$ & $11.45(0.84)$ & $37.91(3.19)$ & & $441.43(14.15)$ & $10.96(0.69)$ & $41.26(3.12)$ \\
13 & $356.44(15.84)$ & $11.91(0.98)$ & $39.87(3.37)$ & & $432.81(13.99)$ & $11.32(0.81)$ & $41.45(3.01)$ \\
14 & $535.82(15.44)$ & $18(1.17)$ & $36.43(3.57)$ & & $526.38(13.03)$ & $17.56(1.06)$ & $50.7(3.06)$ \\
15 & $380.52(14.52)$ & $12.56(1.28)$ & $37.66(3.4)$ & & $447.75(13.16)$ & $9.74(0.74)$ & $43.63(2.97)$ \\
\hline
\end{tabular}


Table B1

\section{Appendix B}

Linear Mixed Model Results

\begin{tabular}{|c|c|c|c|c|c|}
\hline \multirow[b]{2}{*}{ Predictors } & \multicolumn{5}{|c|}{ Reaction Times (ms) } \\
\hline & Estimates & $\mathrm{SE}$ & t-value & & value \\
\hline (Intercept) & 657.60 & 14.62 & 44.99 & $<$ & 0.001 \\
\hline Block [2] & -120.20 & 3.67 & -32.74 & $<$ & 0.001 \\
\hline Block [3] & -154.50 & 3.81 & -40.52 & $<$ & 0.001 \\
\hline Block [4] & -168.50 & 3.92 & -42.96 & $<$ & 0.001 \\
\hline Block [5] & -198.00 & 4.01 & -49.37 & $<$ & 0.001 \\
\hline Block [6] & -207.70 & 4.10 & -50.73 & $<$ & 0.001 \\
\hline Block [7] & -231.20 & 4.08 & -56.64 & $<$ & 0.001 \\
\hline Block [8] & -251.80 & 4.14 & -60.77 & $<$ & 0.001 \\
\hline Block [9] & -249.60 & 4.09 & -61.01 & $<$ & 0.001 \\
\hline Block [10] & -274.00 & 4.06 & -67.45 & $<$ & 0.001 \\
\hline Block [11] & -280.90 & 4.17 & -67.40 & $<$ & 0.001 \\
\hline Block [12] & -295.50 & 4.19 & -70.48 & $<$ & 0.001 \\
\hline Block [13] & -303.80 & 4.18 & -72.71 & $<$ & 0.001 \\
\hline Block [14] & -67.87 & 3.98 & -17.06 & $<$ & 0.001 \\
\hline Block [15] & -261.90 & 4.06 & -64.53 & $<$ & 0.001 \\
\hline MW & -0.41 & 0.09 & -4.52 & $<$ & 0.001 \\
\hline Group [Implicit] & -46.89 & 20.04 & -2.34 & & 0.020 \\
\hline Block [2] x MW & 0.08 & 0.12 & 0.66 & & 0.506 \\
\hline Block [3] x MW & 0.23 & 0.12 & 1.95 & & 0.051 \\
\hline Block [4] x MW & 0.30 & 0.11 & 2.66 & & 0.008 \\
\hline Block [5] x MW & 0.24 & 0.12 & 2.11 & & 0.035 \\
\hline Block [6] x MW & 0.34 & 0.11 & 2.97 & & 0.003 \\
\hline Block [7] x MW & 0.65 & 0.11 & 5.81 & $<$ & 0.001 \\
\hline Block [8] x MW & 0.76 & 0.11 & 6.75 & $<$ & 0.001 \\
\hline Block [9] x MW & 0.67 & 0.11 & 5.94 & $<$ & 0.001 \\
\hline Block [10] x MW & 0.83 & 0.11 & 7.46 & $<$ & 0.001 \\
\hline Block [11] x MW & 0.89 & 0.11 & 8.07 & $<$ & 0.001 \\
\hline Block [12] x MW & 1.02 & 0.11 & 8.94 & $<$ & 0.001 \\
\hline Block [13] x MW & 1.01 & 0.11 & 9.06 & $<$ & 0.001 \\
\hline Block [14] x MW & -0.60 & 0.11 & -5.46 & $<$ & 0.001 \\
\hline Block [15] x MW & 0.52 & 0.11 & 4.64 & $<$ & 0.001 \\
\hline Block [2] x Group [Implicit] & 38.15 & 5.09 & 7.50 & $<$ & 0.001 \\
\hline Block [3] x Group [Implicit] & 54.89 & 5.26 & 10.44 & $<$ & 0.001 \\
\hline Block [4] x Group [Implicit] & 67.68 & 5.39 & 12.55 & $<$ & 0.001 \\
\hline Block [5] x Group [Implicit] & 87.44 & 5.55 & 15.76 & $<$ & 0.001 \\
\hline Block [6] x Group [Implicit] & 84.87 & 5.60 & 15.14 & $<$ & 0.001 \\
\hline Block [7] x Group [Implicit] & 93.85 & 5.67 & 16.55 & $<$ & 0.001 \\
\hline Block [8] x Group [Implicit] & 113.60 & 5.77 & 19.68 & $<$ & 0.001 \\
\hline Block [9] x Group [Implicit] & 98.74 & 5.80 & 17.03 & $<$ & 0.001 \\
\hline Block [10] x Group [Implicit] & 110.60 & 5.70 & 19.42 & $<$ & 0.001 \\
\hline Block [11] x Group [Implicit] & 108.70 & 5.86 & 18.54 & $<$ & 0.001 \\
\hline Block [12] x Group [Implicit] & 138.50 & 5.79 & 23.92 & $<$ & 0.001 \\
\hline Block [13] x Group [Implicit] & 134.20 & 5.84 & 23.00 & $<$ & 0.001 \\
\hline Block [14] x Group [Implicit] & 10.58 & 6.02 & 1.76 & & 0.079 \\
\hline Block [15] x Group [Implicit] & 119.30 & 5.82 & 20.50 & $<$ & 0.001 \\
\hline MW x Group [Implicit] & 0.94 & 0.11 & 8.22 & $<$ & 0.001 \\
\hline
\end{tabular}


Table B1

\section{Appendix B}

Linear Mixed Model Results

\begin{tabular}{lcccc}
\hline & \multicolumn{3}{c}{ Reaction Times (ms) } \\
\cline { 2 - 4 } Predictors & Estimates & SE & t-value & p-value \\
\hline Block [2] x Group [Implicit] x MW & -0.43 & 0.15 & -2.89 & $<.001$ \\
Block [3] x Group [Implicit] x MW & -0.47 & 0.15 & -3.17 & $<$ \\
Block [4] x Group [Implicit] x MW & -0.82 & 0.15 & -5.65 & $<.001$ \\
Block [5] x Group [Implicit] x MW & -0.63 & 0.15 & -4.31 & 0.001 \\
Block [6] x Group [Implicit] x MW & -0.63 & 0.15 & -4.27 & 0.001 \\
Block [7] x Group [Implicit] x MW & -0.78 & 0.14 & -5.43 & 0.001 \\
Block [8] x Group [Implicit] x MW & -1.19 & 0.15 & -8.13 & 0.001 \\
Block [9] x Group [Implicit] x MW & -0.97 & 0.15 & -6.64 & 0.001 \\
Block [10] x Group [Implicit] x MW & -0.96 & 0.14 & -6.67 & $<.001$ \\
Block [11] x Group [Implicit] x MW & -0.87 & 0.14 & -6.07 & $<.001$ \\
Block [12] x Group [Implicit] x MW & -1.53 & 0.15 & -10.46 & $<.001$ \\
Block [13] x Group [Implicit] x MW & -1.36 & 0.15 & -9.29 & $<.001$ \\
Block [14] x Group [Implicit] x MW & -0.22 & 0.14 & -1.51 & $<.001$ \\
Block [15] x Group [Implicit] x MW & -1.20 & 0.15 & -8.20 & 0.132 \\
\hline
\end{tabular}

Random Effects

\begin{tabular}{lc}
\hline$\sigma^{2}$ & 25181.27 \\
$\tau_{\text {00Subject }}$ & 18033.13 \\
ICC & 0.42 \\
$N_{\text {Subject }}$ & 186.00 \\
Observations & 202921 \\
Marginal $R^{2} /$ Conditional $R^{2}$ & $0.085 / 0.467$ \\
\hline
\end{tabular}

Notes: $\mathrm{MW}=$ mind wandering (continuous). The control levels were Block 1 and the Explicit Group. Square brackets indicate the level of the factor contrasted against the control level of each factor. 